\title{
Boaventura de Sousa Santos
}

\section{Plurinationaler Konstitutionalismus und experimenteller Staat in Bolivien und Ecuador}

\section{Perspektiven aus einer Epistemologie des Südens ${ }^{1}$}

Im lateinamerikanischen Kontext geht die Neugründung des Staates in einigen Fällen, wie zum Beispiel in Bolivien und Ecuador, mit der Anerkennung von Plurinationalität einher. Plurinationalität als Prinzip staatlicher Organisation fordert das moderne Staatsverständnis, und damit die Idee der Bürgernation, auf grundlegende Weise heraus. ${ }^{2}$ Eine Bürgernation konstituiert sich aus der Gesamtheit derjenigen Einwohner_innen, welche einen bestimmten geopolitischen Raum bewohnen und vom Staat als Bürger_innen anerkannt werden. Diesem Verständnis zufolge kann also nur eine Nation in einem Staat leben. Die Plurinationalität hingegen erfordert ein Konzept der Nation, welches Nation nicht als Bürgernation, sondern über die gemeinsame Zugehörigkeit zu einer bestimmten Ethnie, Kultur oder Religion konzipiert.

Menschenrechtlich kann Plurinationalität als Anerkennung von kollektiven Rechten sozialer Gruppen gefasst werden. Eine solche ist notwendig, da es in manchen Fällen nicht ausreicht, lediglich die individuellen Rechte der Angehörigen zu schützen, um das Fortbestehen der kulturellen Identität der jeweiligen Gruppe zu gewährleisten oder um ihre soziale Diskriminierung zu beenden. Wie die Existenz einiger plurinationaler Staaten belegt (Kanada, Belgien, Nigeria, Neuseeland etc.), kann die Bürgernation mit verschiedenen kulturellen Nationen innerhalb desselben geopolitischen Raumes (des Staates) koexistieren.

Plurinationalität bedeutet, Selbstregierungs- und Selbstbestimmungsrechte anzuerkennen, ohne dabei notwendigerweise die Gründung unabhängiger Staaten zu unterstützen. Dieses Verständnis teilen die indigenen Völker des lateinamerikanischen Kontinents, und es spiegelt sich in internationalen Menschenrechtsinstrumenten über indigene Völker wider, so zum Beispiel im Übereinkommen 169 der ILO (Übereinkommen über eingeborene und in Stämmen lebende Völker in unabhängigen Ländern [1989/1991]) und der „Erklärung der Vereinten Nationen über die Rechte indigener Völker" vom 7. September 2007 (Resolution 61/295). Die der Plurinationalität zugrunde liegende Idee der Selbstregierung hat viele Facetten und beinhaltet: eine neue Form der staatlichen Institutionenlandschaft, eine neue territoriale Organisation, interkulturelle Demokratie, neue Formen öffentlicher Politiken (Gesundheit, Bildung, soziale Sicherheit) und schließ-

1 Auf Spanisch veröffentlicht in: Santos, Refundación del Estado en América Latina, 2010, S. 81-111. Kürzung und Übersetzung aus dem Spanischen von Almut Schilling-Vacaflor. Die Redaktion hat sich dazu entschlossen, die maskuline Form im Spanischen in den Fällen geschlechterneutral zu übersetzen, in denen sie eine geschlechterübergreifende Gruppe bezeichnet.

2 Das ist auch in einigen Ländern Afrikas der Fall, wo die Plurinationalität als ethnischer Föderalismus begriffen wird, siehe Akiba (Hrsg.), Constitutionalism \& Society in Africa, 2004; Berman/Eyoh/Kymlicka, Ethnicity \& Democracy in Africa, 2004; Keller, Ethnic Federalism, Fiscal Reform, Development and Democracy in Ethiopia, in: African Journal of Political Science 2002, S. 33-34. 
lich die Neugestaltung der öffentlichen Verwaltung, der Bürger_innenbeteiligung, der Dienstleistungen und der öffentlichen Amtsträger_innen. Jede einzelne dieser Facetten fordert die Prämissen heraus, auf denen der moderne Staat aufgebaut ist.

Bevor die einzelnen Bereiche der zu gründenden plurinationalen Staaten im Detail behandelt werden, möchte ich betonen, dass die Anerkennung der Plurinationalität ein grundsätzlich anderes Staatsprojekt bedeutet. Es erfordert nämlich eine Redefinition der Zielbestimmungen für staatliches Handeln und der Beziehungsmuster zwischen Staat und Gesellschaft. Die Anerkennung der nationalen und kulturellen Unterschiede bezieht sich nicht auf ein regelloses Nebeneinander von Weltanschauungen oder auf eine Hybridität bzw. einen Eklektizismus ohne Prinzipien. Im Gegenteil, sie beinhaltet auch interne Hierarchien und Heterogenität: Innerhalb einer Kultur oder Nation können bestimmte Versionen bevorzugt werden, denn kulturelle und nationale Identitäten sind weit davon entfernt, homogen zu sein.

\section{Das Gesellschaftsprojekt}

Die politische Bedeutung der staatlichen Neugründungen erschließt sich aus dem Gesellschaftsprojekt, so wie es in den neuen Verfassungen Boliviens und Ecuadors verankert wurde. ${ }^{3}$ Diese verweisen auf das Prinzip des Buen Vivir (Sumak Kawsay oder Suma Qamaña) als normatives Paradigma der sozialen und wirtschaftlichen Ordnung. ${ }^{4}$ Die neue Verfassung Ecuadors schließt sogar explizit die Rechte der Natur gemäß der andinen Weltanschauung über die Pachamama ein. ${ }^{5}$ Diese Innovationen legen damit fest, dass sich die neuen Staatsprojekte nicht an Wirtschaftsformen orientieren sollen, die kapitalistisch organisiert sind oder vom Ressourcenabbau und Agro-Export abhängen.

Vielmehr privilegieren die neuen Verfassungen ein solidarisches und souveränes Wirtschaftsmodell ${ }^{6}$, das auf einer harmonischen Beziehung mit der Umwelt basiert. Diese wird nach Eduardo Gudynas ${ }^{7}$ nicht mehr als natürliches Kapital verstanden, sondern als Naturerbe. Hierdurch wird eine kapitalistische Wirtschaftsweise zwar nicht aus den Verfassungen ausgeschlossen, aber zumindest wird verhindert, dass allein die globalen kapitalistischen Beziehungen Logik, Richtung und Rhythmus der nationalen Entwicklung bestimmen. Des weiteren unterbinden die neuen Verfassungen nicht, dass die nationale Einheit weiterhin begangen oder sogar intensiviert wird, sondern lediglich, dass im Namen der Einheit die Plurinationalität aberkannt oder abgewertet wird. ${ }^{8}$

Die Verfassungsbestimmungen geben Regierungen, sozialen Bewegungen und Bürger_innen mehr oder weniger eindeutige Orientierungen vor, wie der öffentliche und der private Raum, die politisch-administrativen Institutionen sowie die sozialen und kulturellen Beziehungen organisiert werden sollen. In Bolivien und Ecuador gestalten sich die Regelungen besonders komplex, da die Idee der Plurinationalität von den kulturellen Identitäten ebenso geprägt ist wie von der Idee,

3 Zur Verfassung Ecuadors und dem darin enthaltenen landesweiten Projekt siehe Acosta/Martínez (Hrsg.), El buen vivir: una vía para el desarrollo, 2009; dies., Plurinacionalidad: democracia en la diversidad, 2009; dies., Derechos de la naturaleza: el futuro es ahora, 2009; Walsh, Interculturalidad, Estado, sociedad. Luchas (de)coloniales de nuestra época, 2009.

4 Siehe Art. 275 der Verfassung Ecuadors und Art. 8, 306 und 307 der Verfassung Boliviens.

5 Siehe Art. 71 der Verfassung Ecuadors.

6 Acosta, Siempre más democracia nunca menos: a manera de prologo, in: Acosta/Martínez (Hrsg.), El buen vivir (Fn. 3), S. 20; León, Cambiar la Economía para cambiar la vida, in: ebd., S. 65.

7 Gudynas, Seis puntos clave en ambiente y desarrollo, in: Acosta/Martínez, (Hrsg.), El buen vivir (Fn. 3), S. 39 .

8 Siehe Art. 3 der Verfassung Bolliviens. 
die natürlichen Ressourcen zu kontrollieren. Letztere ist in Bolivien verknüpft

mit einem gesellschaftlichen Kampf um die Verstaatlichung der natürlichen Ressourcen, der mindestens seit der Revolution von 1952 andauert und seit dem so genannten „Wasserkrieg“ (2000) und dem „Gaskrieg“ (2003) wieder ins Zentrum der Konflikte zwischen Staat und Gesellschaft gerückt ist. In diesem Prozess lässt sich beobachten, wie sich eine bolivianische Nation von unten herausbildet, die Zavaleta mit dem Konzept des National-Populären (nacional-popular) umschrieben hat. ${ }^{9}$ Eine solche Idee der bolivianischen Nation ist der Oligarchie fremd, nicht aber der breiten Bevölkerung; daher besteht nicht notwendigerweise ein Widerspruch zwischen einer Verstaatlichung natürlicher Ressourcen und der Plurinationalität. ${ }^{10}$ Indem sie beide Forderungen aufgreift, stützt die indigene Bewegung ihre Handlungen auf die Idee, dass nur ein plurinationaler Staat eine Nation gegenüber dem Ausland bilden kann und gleichzeitig nur der plurinationale Staat eine Nation gegen den internen Kolonialismus hervorbringen kann. Die Pluralität der Nation ist der einzige Weg, um die Nation der Plurinationalität zu bilden.

Aus diesen Gründen bedeutet Plurinationalität nicht, die Nation zu verneinen, sondern anzuerkennen, dass die Bildung der Nation unabgeschlossen ist. Die Polarisierung zwischen der Bürger_innennation und der ethnisch-kulturellen Nation ist somit ein Ausgangspunkt, aber nicht notwendigerweise das Ziel. Der historische Prozess kann zu einem Konzept von Nation führen, das diese Polarisierung überwindet. Die Schaffung von internen „internationalen“ Feldern in den jeweiligen Ländern kann zum Beispiel eine neue Form des transmodernen politischen Experimentalismus darstellen.

\section{Die neue Institutionenlandschaft}

Die Plurinationalität impliziert das Ende der institutionellen Homogenität des Staates. Die zu schaffende Heterogenität kann interner oder externer Natur sein. Sie ist immer dann intern, wenn innerhalb ein- und derselben Institution institutionelle Zugehörigkeiten eingerichtet sind, die den kollektiven Rechten entsprechen. Sie ist extern, wenn zwei parallele und/oder asymmetrische Institutionen die Anerkennung der Unterschiede garantieren. Es gibt folglich zwei unterschiedliche Formen der institutionellen Anerkennung der Unterschiede, die von der Plurinationalität abgeleitet werden können: jene, die (gemeinsam) innerhalb der gleichen Institutionen eingebettet werden, und jene, die (zwei) unterschiedliche Institutionen voraussetzen.

Ein Beispiel für die gemeinsamen Institutionen ist die kürzlich gewählte Plurinationale Legislative Versammlung von Bolivien, in der sieben Sitze für ethnische Minderheiten reserviert sind, welche wiederum nach eigenen Normen und Prozeduren gewählt werden. ${ }^{11}$ Nicht freie und allgemeine Wahlen von Repräsentant_innen verschiedener Kulturen oder Nationen machen daher den plurinationalen Charakter der Legislative aus, sondern die Anerkennung unterschiedlicher Kriterien der politischen Repräsentation dieser Kulturen oder Nationen. Was heute aus der Perspektive der liberalen politischen Kultur absurd erscheint,

9 Zavaleta, Lo nacional-popular en Bolivia, 1986.

10 Luis Tapia merkt in diesem Sinne an, dass „die Kritik an der Idee eines homogenen Staates mit der Forderung nach Verstaatlichungen in keinem Widerspruch zueinander [...]“ steht; Tapia, Una reflexión sobre la idea de un estado plurinacional, 2008, S. 67.

11 Im Zuge der politischen Verhandlungen über das neue Wahlsystem wurde die Anzahl reservierter Sitze eingeschränkt, und es entstand eine Asymmetrie zugunsten des eurozentrischen Systems der politischen Repräsentanz; das bedeutet, dass die Kriterien zur Wahl der Repräsentant_innen weniger plurinational sind als die repräsentierte Plurinationalität. 
kann morgen als Praxis der Gleichheit in der Differenz akzeptiert sein. Es ist denkbar, dass die Unterschiede zwischen den verschiedenen Repräsentationsformen im Laufe der Zeit geringer werden, wenn auch nicht hinsichtlich der ihnen zugrunde liegenden Prinzipien, so doch zumindest in den politischen Praktiken, die sich aus ihnen ableiten. Die Prozesse der Hybridisierung sollten anhand des erreichten Ausmaßes der Inklusion und Partizipation bewertet werden.

Ein weiteres Beispiel für Plurinationalität findet sich in den neu zu bildenden Verfassungsgerichten. Diese Institutionen erfüllen eine zentrale Funktion im plurinationalen Staat, denn sie sind dafür zuständig, einige der kompliziertesten Konflikte, die sich aus der Koexistenz verschiedener Nationen in ein und demselben geopolitischen Raum ergeben, zu lösen. Damit das Verfassungsgericht tatsächlich plurinational ist, reicht es nicht aus, dass Repräsentant_innen verschiedener Nationalitäten darin vertreten sind. Vielmehr sollte der Prozess seiner Zusammensetzung selbst notwendigerweise plurinational sein. ${ }^{12} \mathrm{Im}$ Falle Ecuadors wurde das vorausgehende Verfassungstribunal in das neue Verfassungsgericht umgewandelt und mit zusätzlichen Kompetenzen ausgestattet. Es arbeitet so lange in der bisher bestehenden Zusammensetzung, bis die Mechanismen der Richter_innenernennung festgelegt wurden. Das Verfassungsgericht (bisher als „Verfassungsgericht für die Übergangsperiode“ bezeichnet) hat seine neuen Aufgaben in Gänze übernommen. ${ }^{13}$

Ein drittes Beispiel einer zentralen gemeinsamen Institution für die Konstruktion der neuen bolivianischen Demokratie ist die plurinationale Wahlbehörde (Art. 245 ff. der Verfassung), das vierte staatliche Organ neben der Exekutive, der Legislative und der Judikative. Es soll die Prozesse der politischen Repräsentation beobachten und kontrollieren, eine Aufgabe, die angesichts der neuen in der Verfassung festgeschriebenen hohen Komplexität der politischen Repräsentation eine große Herausforderung darstellt. Sie beinhaltet nicht nur verschiedene Ausformungen der repräsentativen Demokratie (nationale, Departement- und Gemeinde-Ebene), sondern auch verschiedene Formen der Organisation von Interessen (Parteien und zivilgesellschaftliche Organisationen) sowie unterschiedliche Formen der Demokratie (repräsentativ, partizipativ und gemeinschaftlich). Außerdem wurde dem Wahlorgan auch die Aufgabe übertragen, die interne Demokratie der politischen Organisationen zu überprüfen, inklusive der Normen und Verfahren der indigenen Völker und bäuerlichen Gemeinschaften. Bereits die Zusammensetzung der Wahlbehörde soll die plurinationale Natur des Staates ausdrücken. Deswegen legt die Verfassung die obligatorische Präsenz von Repräsentant_innen indigener und bäuerlicher Nationen, Völker und Gemeinschaften im Wahlorgan fest (mindestens zwei auf nationaler und eine auf Departement-Ebene).

José Luis Exeni, der bis zum 1. Mai 2009 Präsident des nationalen Wahlgerichtshofs war, wies darauf hin, dass die demokratische Konstruktion des neuen Staatsmodells nicht einfach sein würde, weil es die Realität der 36 indigenen und bäuerlichen Völker, Nationen und Gemeinschaften (sowie jene der interkulturellen und afrobolivianischen Gemeinschaften) widerspiegeln müsse. ${ }^{14}$ Die Verfassung schreibt dem neuen Staatsmodell elf Adjektiv-Attribute zu: einheitlich, sozial,

13 Siehe beispielsweise das Gerichtsurteil über die indigene Universität „Amwtay Wasi“, Fall N 0027-09AN vom 9. Dezember 2009.

14 Exeni, Miradas al Título IV de la Constitución Política del Estado. Un Órgano Electoral para la demodiversidad, 2010. 
rechtsstaatlich, plurinational, gemeinschaftlich, frei, unabhängig, souverän, de-

mokratisch, interkulturell und dezentralisiert mit Autonomien. In diesem $\mathrm{Zu}$ sammenhang stellt sich Exeni unter anderem folgende Fragen: „Wie kann eine Demokratie mit großer Intensität in Bolivien geschaffen werden, welche dem Prinzip der geteilten Autorität und der Herausforderung der Demo-Diversität gerecht wird? Wie kann das perverse lateinamerikanische Dreieck bestehend aus Wahldemokratie, sozialer Ungleichheit und Armut überwunden werden? Was bedeutet der Horizont des Verfassungs-Experimentalismus für die Struktur und die Reichweite des politischen Regimes und im Speziellen für die bolivianische Wahlbehörde? Konkret: Auf welche Prinzipien und Normen sollen die Gesetze des Wahlsystems aufbauen, die von der neuen plurinationalen legislativen Versammlung ausgearbeitet werden?". 15

Ein Beispiel für duale Institutionen hingegen sind die territorialen Autonomien (siehe unten). Die Verfassung Boliviens verankert im Abschnitt zur territorialen Struktur und Organisation des Staates vier parallele Typen von Autonomien: departementale, regionale, kommunale und indigen-bäuerliche. Die Verfassung Ecuadors wiederum erkennt fünf autonome Regierungen an (Art. 238) und sieht die Schaffung indigener und plurikultureller territorialer Wahlkreise mit speziellen Wahlregimen vor (Art. 242). Die vorgesehenen Autonomien lassen sich in zwei Gruppen unterteilen, denn ausschließlich die indigene (oder im Fall Ecuadors die plurikulturelle) Autonomie kann Rechtspluralismus für sich geltend machen. Obwohl alle unterschiedlichen Formen der Autonomie bestimmte legislativ-normative Kompetenzen besitzen, ist lediglich die indigene im Rahmen ihres Selbstbestimmungsrechtes rechtlich autonom. Diese Autonomie leitet sich aus der verfassungsrechtlichen Anerkennung ihrer traditionellen Rechtsprechung ab. Der Rechtspluralismus (traditionelles Recht auf der einen Seite und eurozentrisches Recht auf der anderen) ist ein weiterer Fall der institutionellen Dualität, wie wir im folgenden Abschnitt sehen werden.

\section{Der Rechtspluralismus}

Die moderne liberale Symmetrie - der gesamte Staat ist ein Rechtsstaat, und Recht ist immer staatliches Recht - ist eine der großen Innovationen der westlichen Moderne. Diese Symmetrie ist problematisch, nicht zuletzt weil sie das vielfältige nicht-staatliche Recht in den Gesellschaften verkennt.

Der plurinationale Konstitutionalismus bricht mit diesem Paradigma, weil für ihn die Einheit des Rechtssystems nicht dessen Einheitlichkeit voraussetzt. Im Rahmen der Plurinationalität erhält die verfassungsrechtliche Anerkennung des traditionellen indigenen Rechts eine zusätzliche Bedeutung, denn hierdurch wird die Interkulturalität gefördert und die Selbstregierung der indigen-bäuerlichen Gemeinschaften gefestigt. Die zwei oder drei existierenden Rechtssysteme - eurozentrisch, indozentrisch und, in einigen Ländern oder Situationen, afrozentrisch - sind zwar autonom, aber nicht unvermittelbar. Die Beziehungen zwischen ihnen stellen die beteiligten Akteur_innen in jedem Fall vor eine Herausforderung: Nach zwei Jahrhunderten einer vermeintlichen rechtlichen Einheitlichkeit wird es für Bürger_innen, soziale Organisationen, politische Akteur_innen, öffentliche Bedienstete, Anwält_innen und Richter_innen nicht einfach sein, ein umfassenderes Rechtskonzept anzunehmen, welches durch die Anerkennung pluraler Rechtsordnungen das Recht teilweise vom Staat entkoppelt und es an das Leben und die Kultur der Völker rückbindet. 
Verschiedene Formen der Legalität werden in Zukunft offiziell koexistieren und miteinander in Konflikt treten. Der Unterschied zwischen diesen Ordnungen lässt sich am besten anhand der gesellschaftlichen Beziehungsformen aufzeigen, die sich an den Schnittstellen zwischen den verschiedenen kulturellen Universen ergeben und welche von den unterschiedlichen rechtlichen Paradigmen jeweils bevorzugt bzw. abgelehnt werden. ${ }^{16}$ Ich unterscheide vier solcher Beziehungsformen: Gewalt, Koexistenz, Versöhnung und Zusammenleben.

Die Gewalt ist die Art des Zusammentreffens, in der die dominante Kultur eine absolute Kontrolle über die Kontaktzone beansprucht und sich dazu legitimiert fühlt, die subalterne Kultur zu unterdrücken, zu marginalisieren oder sogar zu zerstören. Koexistenz ist das typische Beziehungsmuster der kulturellen Apartheid, in der sich die verschiedenen Rechtskulturen getrennt voneinander und einer rigiden Hierarchie folgend entwickeln. Kontakte, Vermischungen und Hybridisierung werden starr vermieden oder komplett verboten. In diesem Fall werden parallele Rechtssysteme gestattet, aber mit vollkommen asymmetrischem Status, was sowohl die Hierarchisierung als auch die Trennung zwischen beiden Systemen garantiert. Die Versöhnung ist das Fundament der restaurativen Gerechtigkeit, welche darauf abzielt, die Verletzungen der Vergangenheit zu heilen. Sie ist eine Beziehungsform, die sich stärker an der Vergangenheit als an der $\mathrm{Zu}$ kunft orientiert. Daher bestehen die Ungleichheiten der Vergangenheit häufig unter einem neuen Deckmantel fort. Die Rechtssysteme kommunizieren miteinander nach Regeln, die beispielsweise das kommunale oder indigene Recht als Relikt der Vergangenheit konzipieren, welches überwunden werden müsse. Das Paradigma des Zusammenlebens ist gewissermaßen eine Versöhnung, die auf die zukünftige Entwicklung hin ausgerichtet ist. Hier wurden vergangene Konflikte auf eine Weise bewältigt, die eine auf gleichwertigem Austausch und geteilter Autorität beruhende Beziehung ermöglicht. Die verschiedenen Rechtsuniversen werden als alternative Zukunftsvisionen behandelt, und bei Konflikten zwischen ihnen werden diese nach gemeinsam erarbeiteten Verfassungsregeln gelöst. Lediglich dieses letzte Beziehungsmuster respektiert das Prinzip der Plurinationalität.

Ein solches Zusammenleben von Rechtssystemen offiziell anzuerkennen, bedeutet Veränderungen sowohl für das (vielfältige) traditionelle, als auch für das eurozentrische Recht. Die verfassungsrechtlichen Grenzen der indigenen Rechtsprechungen (personelle, materielle und territoriale Grenzen) reichen jedoch nicht aus, um die Konflikte auszuräumen, die nicht mehr in einem normativen Rahmen der Legalität, sondern in jenem der Inter-Legalität entstehen. Die Lösung von solchen Konflikten wird immer prekär, riskant und vorläufig sein, denn sie verpflichtet zur interkulturellen Übersetzung (Was ist ein gerechtes Verfahren im traditionellen Recht? Kann ein Traum das Fundament einer legitimen Verteidigung darstellen?). Dennoch ist dies der Weg der beidseitigen Würde und des gegenseitigen Respekts, der Weg der Dekolonisierung. Innerhalb und außerhalb des Rechtsfeldes werden Institutionen und Praktiken der Mediation aufkommen, und die wichtigste Institution diesbezüglich wird in Bolivien und Ecuador der Verfassungsgerichtshof sein.

In ihrem Artikel 30 etabliert die bolivianische Verfassung eine umfassende Liste an Rechten der indigenen und bäuerlichen Nationen, Völker und Gemeinschaften. Dies ist der erstmalige verfassungsrechtliche Ausdruck der Korrespondenz zwischen der großen Bevölkerungsmehrheit und dem politischen Protagonismus der indigenen Völker. Unter diesen Rechten ist es jenes der eigenen Rechtspre- 
chung, das in den Artikeln 190, 191 und 192 ausbuchstabiert wird. In der Verfassung von Ecuador sind ebenfalls die Rechte der indigenen Völker (Art. 57) und der indigenen Rechtsprechung verankert (Art. 171).

Der Vergleich beider Verfassungen verdeutlicht die Ähnlichkeiten zwischen ihnen. In beiden Fällen soll die indigene Rechtsprechung die in der Verfassung verankerten Rechte und Garantien respektieren. In Ecuador findet sich außerdem die Anforderung der gleichwertigen Partizipation der Frauen, die bereits im Forderungskatalog des Bündnisses der indigenen Nationalitäten Ecuadors CON$\mathrm{AIE}^{17}$ für die neue Verfassung enthalten war. Die personelle, materielle und territoriale Gültigkeit der indigenen Justiz weist ebenso Ähnlichkeiten auf. Die indigene Justiz wird ausschließlich auf die indigene Bevölkerung angewandt, was das Problem hervorruft, dass sie nicht anwendbar ist, wenn sowohl indigene als auch nicht-indigene Personen in Konflikte involviert sind. Außerdem ist die indigene Rechtsprechung auf indigene Territorien beschränkt, was zwei weitere Probleme mit sich bringt. Erstens ist die Abgrenzung dieser Territorien in vielen Fällen unklar. Und zweitens finden viele Konflikte zwischen Indigenen außerhalb ihrer Territorien statt. Gemäß der Verfassung Ecuadors kann die indigene Rechtsprechung jedoch nur auf interne Konflikte angewandt werden. Die bolivianische Formulierung geht hier weiter und legt explizit fest: „[die indigene Rechtsprechung] wird auf Rechtsbeziehungen oder -tatsachen angewandt, die unter die indigene Jurisdiktion fallen oder diese betreffen." (Art. 191 Abs. 2 Satz 3 bolivianische Verfassung). Das bedeutet, dass bei Verletzung indigener Rechtsgüter die indigene Rechtsprechung auch außerhalb des indigenen Territoriums anwendbar ist (mit Auswirkungen innerhalb des Territoriums). Hinsichtlich der materiellen Gültigkeit legt die bolivianische Verfassung fest, dass die indigene Rechtsprechung indigene Angelegenheiten „in Übereinstimmung mit dem Gesetz der Kooperation und Koordination der Justiz“ (Art. 191 Abs. 2 Satz 2 bolivianische Verfassung) behandelt. Bis zur Verabschiedung der neuen Gesetze ist die materielle Kompetenz der indigenen Justiz, ebenso wie in Ecuador, allgemeiner Natur. Schließlich sehen beide Verfassungen vor, Mechanismen der Kooperation und Koordination zwischen der indigenen und der ordentlichen Justiz zu schaffen.

\section{Die neue territoriale Aufteilung}

Der moderne liberale Staat ist der politische Ausdruck der modernen Entkontextualisierung der Lebenswelt. In der Wissenschaft oder im Recht ist das Universelle das unabhängig vom Kontext Gültige. Die Glaubwürdigkeit des Universellen wird gestärkt durch die Metaphern der Homogenität, der Gleichheit, der Atomisierung und der Indifferenz. Die beiden wichtigsten Metaphern sind jedoch jene der Zivilgesellschaft und des nationalen Territoriums. Die erste macht alle Bevölkerungsgruppen, die zweite den geopolitischen Raum gleich. Beide stehen miteinander in Beziehung, denn nur nicht unterschiedene Menschen können im nicht unterschiedenen Raum leben.

Diese Konstruktion ist ebenso hegemonial wie willkürlich, und sie lenkt die soziologische, politische und kulturelle Realität in ihre politisch tolerierten Grenzen. Je weiter die Realität von diesen Grenzen entfernt ist, desto autoritärer und ausschließender wird die liberale Demokratie. Der plurinationale Konstitutionalismus bricht radikal mit dieser ideologischen Konstruktion. Einerseits setzt er, indem er die Existenz von Gemeinschaften, Völkern, Nationen und Natio- 
nalitäten anerkennt, die Zivilgesellschaft wieder in ihren Kontext. Andererseits macht er das nationale Territorium zu einem Raum der Einheit und Integrität, der die Beziehungen zwischen verschiedenen geopolitischen und geokulturellen Territorien regelt. Dabei sind die Verfassungsprinzipien der Einheit in der Diversität und der Integrität bei gleichzeitiger Anerkennung asymmetrischer Autonomien maßgeblich.

Die Asymmetrie zwischen Autonomien gründet auf zwei Faktoren: 1) die politisch-administrative Dimension der Dezentralisierung und der regionalen Gerechtigkeit und 2) den politisch-kulturellen Faktor der Plurinationalität und der historischen Justiz (im Fall der indigen-bäuerlichen Autonomien). Im letzteren Fall hat die territoriale Autonomie eine spezifische Begründung und historischkulturelle Dichte. Da diese Einheiten bereits vor dem modernen Staat existierten, sind es nicht die Bewohner_innen des Territoriums, die diese Autonomien begründen müssen, sondern der Staat, der die Grenzen, die er den Autonomien im Namen des nationalen Interesses auferlegt, rechtfertigen muss (paradoxerweise ist ein Teil dieses nationalen Interesses wiederum die Förderung der Autonomien).

Die Departement- und Gemeinde-Wahlen Boliviens am 4. April 2010 waren ein wichtiger Schritt für die Konstruktion des plurinationalen Staates und insbesondere für die departementalen, regionalen, kommunalen und indigenen Autonomien. Zum ersten Mal wurden neben der Gemeinderegierung auch departementale Gouverneure und Versammlungen gewählt. Der plurinationale Charakter des Staates zeigte sich in der direkten Wahl von 23 Departement-Abgeordneten, die Minderheiten in den jeweiligen Departements angehören. Es handelt sich um den dritten konkreten Fortschritt nach der Wahl im Dezember 2009 (durch anonyme Wahlen, nicht eigene Normen und Verfahren) von sieben indigenen Abgeordneten in speziellen Wahlkreisen und der Zustimmung im Rahmen eines Referendums zur Schaffung von elf indigenen Autonomien auf Gemeinde-Ebene. Die indigenen Völker und Nationen registrierten damit vor der Wahlbehörde ihre eigenen Normen und Verfahren, nach denen ihre Abgeordneten gewählt, ernannt oder nominiert wurden. Die Diversität dieser eigenen Normen und Verfahren für die Wahl oder Bestimmung ihrer Abgeordneten ist eine Bekräftigung der Demo-Diversität und der interkulturellen Demokratie.

Die Termini, nach denen diese Autonomien gegründet werden, sind im Weiteren auch entscheidend, um die Art der Kontrolle, die der Zentralstaat legitimerweise innerhalb der autonomen Territorien ausüben kann, zu bestimmen. Es ist bekannt, dass die indigenen Autonomien durch das internationale Recht gestützt werden, ${ }^{18}$ welches unter anderem die Kontrolle der natürlichen Ressourcen und die diesbezügliche Teilhabe an den Vorteilen aus ihrer Nutzung regelt. Diese internationalen Normen wurden von den plurinationalen Staaten anerkannt. Sie betreffen einen umkämpften Aspekt indigener Autonomien, denn gerade in den betroffenen Territorien befindet sich ein Großteil der natürlichen Ressourcen. Dabei geht es nicht um eine Ethnisierung des Reichtums (für die Ethnisierung der Armut gibt es ausreichend historische Beweise), sondern um neue und inklusivere Kriterien für nationale Solidarität. Die Forderung nach historischer Gerechtigkeit ist nichts anderes als die öffentliche Kritik an der brutalen Abwesenheit nationaler Solidarität während der letzten Jahrhunderte. Wie kann die Tatsache erklärt werden, dass die Ärmsten in den Territorien mit dem größten Reichtum leben? Die Solidarität muss auf der Basis postkolonialer Prinzipien neu 
geschaffen werden: Affirmative Aktivitäten zugunsten der indigen-bäuerlichen

Gemeinschaften sind Grundbedingung für eine plurinationale Solidarität.

\section{Neue Organisation des Staates und neue Form der Planung}

Alle bisher angeführten Veränderungen, die sich aus der Idee des plurinationalen Staates ergeben, verpflichten zu einer neuen Organisation des Staates. Diese betrifft alle politischen und administrativ-bürokratischen Institutionen der öffentlichen Verwaltung und Planung. Es ist dieser Bereich, der darüber Auskunft gibt, in welchem Ausmaß das Prinzip der Plurinationalität im neuen politischen Pakt vorhanden ist und inwieweit dieses Prinzip relativiert und mit anderen Prinzipien verknüpft wird.

Ein systematischer Verfassungsvergleich zeigt, dass die Plurinationalität im bolivianischen Fall viel verbindlicher ist als im Fall Ecuadors. Dies erklärt sich aus den politischen Prozessen, aus denen die neuen Verfassungen basieren. In Ecuador steht das Prinzip der Plurinationalität in einer ständigen Spannung mit dem Prinzip der Bürger_innenbeteiligung, welches tatsächlich die stärkste übergreifende Bedingung der neuen ecuadorianischen Verfassungsarchitektur darstellt. Die Verfassung Boliviens etabliert vier Organe: Legislative, Exekutive, Justiz und Wahlbehörde; die Legislative, die als Plurinationale Legislative Versammlung bezeichnet wird, beinhaltet die Repräsentation von speziellen indigenen Wahlkreisen (Art. 146). Die Verfassung von Ecuador definiert die Organisation des Staates als „Partizipation und Organisation der Macht“ (Titel 4); ebenfalls etabliert sie die „Partizipation in Demokratie“ (Kap. 1) als zentrale Orientierung und bestimmt zu diesem Zweck fünf „Funktionen“ des Staates - Legislative; Exekutive; Justiz und indigene Justiz; Transparenz und soziale Kontrolle; Wahlen. Doch die legislative Funktion, ausgeübt durch die nationale Versammlung, erkennt die direkte indigene Repräsentation nicht an, obwohl dies von der CONAIE gefordert wurde.

Theoretisch müsste es keine Spannungen zwischen den Prinzipien der Plurinationalität und der Partizipation geben. Im Gegenteil, die Plurinationalität beinhaltet die Idee von weit fortgeschrittenen und komplexen Formen der Partizipation. Neben der liberal-republikanischen Bürger_innenbeteiligung erkennt sie die Partizipation von Völkern oder Nationalitäten an. Die Verknüpfung der beiden Prinzipien bzw. die mögliche Spannung zwischen ihnen betrifft die Organisation und Funktionalität des Staates auf verschiedenen Ebenen. Um dies zu illustrieren, möchte ich den Fall der öffentlichen Verwaltung und Planung heranziehen. In Bolivien ist das Gesetz der Öffentlichen Verwaltung des Plurinationalen Staates in Vorbereitung. Zwei zentrale Ideen sollen hier hervorgehoben werden. Erstens verlangt die Konstruktion des neuen Staatsmodells den Abbau des Kolonialismus, der sich in den rassistischen Praktiken und der übermäßigen Bürokratisierung der öffentlichen Verwaltung audrückt. Zweitens benötigen die öffentlichen Politiken eine zyklische Planung, die Aktivitäten und Resultate mit den Temporalitäten der unterschiedlichen öko-kulturellen und administrativen Räume im Land in Übereinstimmung bringt und mit dem Ziel des Buen Vivir verbindet. Der Entwurf des Gesetzes über öffentliche Verwaltung (Februar 2010) besagt in Art. 1, dass

„die interkulturelle und kommunale plurinationale öffentliche Verwaltung die Gesamtheit der integralen und komplementären Prozesse darstellt, die die partizipativen öffentlichen Politiken und Strukturen mit der holistischen und kommunalen Kosmovision verbinden, die den indigenen und bäuerlichen Nationen und Völkern eigen ist". 
In Ecuador werden die Herausforderungen der Partizipation in ähnlicher Intensität erlebt, jedoch mit einem anderen Fokus. Die Bürger_innenbeteiligung ist in diesem Fall die zentrale Achse der Suche nach einer partizipativen Planung. Gleichzeitig geht es um Plurinationalität. Der nationale Entwicklungsplan trug sogar den Namen „Nationaler Plan für das Buen Vivir, 2009-2013: die Schaffung eines Plurinationalen und Interkulturellen Staates“. ${ }^{19}$ Doch die Idee des Buen Vivir findet sich nicht eingebettet in partizipative Praktiken im Rahmen der Plurinationalität, es gibt hierbei also keinen besonderen Fokus auf die Praktiken und Konzeptionen der indigenen Völker. Die Spannungen innerhalb der ecuadorianischen Regierung lassen sich daran erkennen, dass die politische Logik des Entwicklungsplans konterkariert wird von der Praxis diejenigen Gesetze, die die indigenen Völker betreffen, ohne vorherige Konsultation zu verabschieden. Dies widerspricht nicht nur der Verfassung, sondern auch der ILO-Konvention 169 und der UN-Erklärung über die Rechte indigener Völker.

In jedem Fall ist die Neugründung des Staates nach den Prinzipien der Plurinationalität, Interkulturalität und demokratischen Partizipation ein langfristiger und komplexer politischer Prozess. Für einen langen Zeitraum werden die Prinzipien und Diskurse den Praktiken weit voraus sein. An der Distanz zwischen ihnen wird sich die Intensität der Demokratisierung der Demokratie messen lassen müssen.

\section{Die interkulturelle Demokratie}

Plurinationalität ist die Anerkennung dessen, dass Interkulturalität nicht aus einem freiwilligen Akt der historischen Reue der Privilegierten entsteht. Sie ist vielmehr das Resultat eines politischen Konsenses zwischen verschiedenen ethnisch-kulturellen Gruppen, der trotz einer von Gewalt gekennzeichneten gemeinsamen Geschichte ein Möglichkeitsfenster für eine andere Zukunft öffnet. Im Rahmen der Plurinationalität findet Interkulturalität allein in der interkulturellen Demokratie ihren Ausdruck.

Unter dem Begriff der interkulturellen Demokratie im lateinamerikanischen Kontinent verstehe ich 1) die Koexistenz verschiedener Formen der demokratischen Deliberation, von der individuellen Wahl bis zur Konsensentscheidung; von Wahlen zur Bestimmung der Autoritäten nach dem Rotationsprinzip bzw. dem „gehorchend führen“ (mandar obedeciendo); vom Wettkampf um Ämter zur Verpflichtung, diese auszuüben (diese Koexistenz bezeichne ich als DemoDiversität), 2) verschiedene Kriterien der demokratischen Repräsentation, 3) Anerkennung der Kollektivrechte der Völker als Bedingung zur effektiven Ausübung von Individualrechten, 4) Anerkennung umfassender fundamentaler Rechte: Recht auf Wasser, Land, Nahrungssicherheit, natürliche Ressourcen, Biodiversität, Wälder und traditionelles Wissen und 5) Bildung mit dem Ziel, gegenseitige kulturelle Anerkennung zu fördern.

Die Verfassungen Boliviens und Ecuadors beinhalten bereits die Idee der interkulturellen Demokratie. Beispielsweise erkennt die bolivianische Verfassung drei verschiedene Demokratieformen an: die repräsentative, die partizipative und die gemeinschaftliche (vgl. Art. 11 der Verfassung Boliviens). Es handelt sich dabei um eine der fortschrittlichsten verfassungsrechtlichen Formulierungen weltweit. Die Departement- und Gemeindewahlen am 4. April 2010 haben die interkulturelle Demokratie auf beeindruckende Weise bestätigt. Diese Form der Demo- 
kratie wirft zwei Probleme auf, die verdeutlichen, wie sie sich von der eurozentrischen Demokratietradition unterscheidet.

Das erste Problem ist, Deliberationen, die dem Konsens- und Einstimmigkeitsprinzip folgen, oder demokratische Wahlen mit dem Rotationsprinzip zu vereinbaren. Diese Schwierigkeiten sollten intern diskutiert werden, denn wie wir wissen, sind Gemeinschaften weder kulturell noch politisch homogen, und Gender- oder Generationsunterschiede können in den Debatten entscheidend sein. Das zweite Problem, das ebenso wie das erste üblicherweise aus einer monokulturellen Kritik an der Interkulturalität heraus aufgeworfen wird, besteht darin, dass die indigenen Gemeinschaften undemokratische Enklaven darstellen, in denen Frauen systematisch diskriminiert werden. Auch wird häufig kritisiert, dass durch die Anerkennung der Kollektivrechte in der interkulturellen Demokratie die Individualrechte verletzt würden. Diese Kritikpunkte sind relevant, um die Funktionsweise der interkulturellen Demokratie zu verbessern. Den indigenen Frauen wird jedoch heutzutage immer klarer, dass das Buen Vivir zu Hause anfängt, und sie gehören bereits zu den Protagonistinnen der aktivsten und innovativsten Frauenbewegungen des Kontinents. ${ }^{20}$

Die Kollektivrechte kollidieren nicht notwendigerweise mit den individuellen Rechten. Es gibt verschiedene Arten von Kollektivrechten: abgeleitete und ursprüngliche. Wenn beispielsweise Arbeiter_innen ihre Gewerkschaft gründen und die Verteidigung ihrer individuellen Arbeitsrechte an diese delegieren, ist diese Gewerkschaft dazu berechtigt, ihre Mitglieder zu repräsentieren. In diesem Fall gibt es keinen Konflikt zwischen verschiedenen Rechtstypen. Die ursprünglichen Kollektivrechte wiederum sind jene der Gemeinschaften und können daher unter bestimmten Umständen mit den Individualrechten in Konflikt treten. Für diesen Fall sollte es eigene Instanzen der Konfliktlösung geben, welche häufig interkulturelle Übersetzungsarbeit benötigt. Beispielsweise ist es nicht legitim, ausgehend von einem eurozentrischen Rechtsverständnis zu entscheiden, ob ein Fall der indigenen Justiz gegen das Recht auf ein gerechtes Verfahren verstoßen hat (so wäre ja etwa die Abwesenheit von professionellen Anwält_innen in der indigenen Justiz schon per Definition ein solcher Verstoß). Im Gegenteil, um ein gerechtes Verfahren herzustellen ist es notwendig, interkulturelle Kriterien zu formulieren, die Äquivalente zwischen unterschiedlichen Formen zur Herstellung eines gerechten Verfahrens festlegen. Die interkulturelle Übersetzung müsste außerdem berücksichtigen, dass die Beziehung zwischen Rechten und Pflichten keine universelle Konstante darstellt; sie variiert je nach Rechtskultur. Im indigenen Recht ist die Gemeinschaft in stärkerem Ausmaß eine Gemeinschaft von Pflichten als von Rechten; wer die Pflichten nicht akzeptiert, hat daher auch keine Rechte. ${ }^{21}$

Hinzuzufügen ist, dass die ursprünglichen Kollektivrechte auf zwei Arten ausgeübt werden können. Individuell, z.B. wenn ein Sikh seinen Turban trägt, oder kollektiv, wenn es sich beispielsweise um das Recht auf Selbstbestimmung oder Selbstregierung handelt. Die verschiedenen Kollektivrechte können strukturelle oder historische Ungerechtigkeiten verringern, und sie begründen notwendige affirmative Aktionen, um Gemeinschaften oder Völker von der systematischen Unterdrückung zu befreien oder um ihr Überleben als Kollektive zu garantieren. 
Im lateinamerikanischen Kontext ist die Mestizisierung ein Produkt des Kolonialismus und der indigenistischen Politik. Auch wenn dabei die indigene Identität anerkannt wurde, wurde Fortschritt immer mit der eurozentrischen Akkulturalisierung identifiziert. Vor diesem Hintergrund kann der indigene Kampf um die Plurinationalität der Idee der Mestizisierung nur feindlich gegenübertreten. Dennoch gibt es in diesem Kontext komplexe Konstellationen, die nicht ignoriert werden sollten, wie z.B. Mestiz_innen, die sich gleichzeitig als Indigene identifizieren, oder Mestiz_innen, die als Alliierte der Indigenen agieren und die Idee der Plurinationalität verteidigen. Könnte der indigene Protagonismus unter der Fahne der Plurinationalität dazu führen, die Ziele einer großen Bevölkerungsmehrheit, die sich als Mestiz_innen definiert und ein wichtiger Teil aktueller Transformationsprozesse ist, in den Hintergrund zu drängen oder zu marginalisieren?

Aus einer anderen Perspektive ist es offensichtlich, dass das Konzept der Mestizisierung historisch ganz unterschiedliche Bedeutungen gehabt hat. ${ }^{22}$ Die soziale Kategorie des/der „Mestiz_in“ verdeckt enorme soziale Differenzen, nämlich, dass neben dem/der weißen Mestiz_in immer der/die Indio-Mestiz_in existierte und dass ihre Beziehungen häufig die koloniale und rassistische Differenz reproduzierten. Eine andere Dimension der Komplexität dieses Themas hat weniger mit der Vergangenheit als mit der Zukunft zu tun. Die Plurinationalität schafft den Rahmen für neue Typen von interethnischen und interkulturellen Beziehungen, von denen neue empirische, kulturelle und konzeptuelle Hybridisierungen ausgehen. Wie kann, ausgehend von der Idee der Plurinationalität, Mestizisierung neu verstanden werden?

Das Konzept der Interkulturalität verlangt danach, die Gemeinsamkeiten verschiedener Kulturen zu bestimmen, so dass das „Inter“ einen Sinn erhält. Die Unterscheidung zwischen Intrakulturalität und Interkulturalität ist komplex, denn sie ist das Resultat einer sich verändernden gesellschaftlichen Konstruktion. Die Transformation der Interkulturalität in Bolivien und Ecuador ist ein historisch doppelt komplexer Prozess. Erstens gilt es, vertikale Beziehungen zwischen Kulturen in horizontale Beziehungen zu verwandeln. Zweitens kann sie nicht zum Relativismus führen, sobald sie in einem bestimmten verfassungsrechtlichen Rahmen stattfindet.

Noch wichtiger als die Kenntnis des gemeinsamen Fundaments ist die Identifizierung der politisch-kulturellen Bewegung, die schrittweise Mentalitäten und Subjekte verändert, und zwar im Sinne einer Anerkennung von Gleichheit/ Äquivalenz/Komplementarität/Reziprozität zwischen Unterschieden. Erst dann kann ein bereichernder Dialog entstehen, denn dies ist der Moment, bei dem die Unvollständigkeit jeder einzelnen Kultur ersichtlich wird; jede Kultur ist auf ihre Weise problematisch und unfähig dazu, allein auf den Wunsch der Völker zu antworten, eine tatsächlich inklusive Gesellschaft zu konstruieren. Die Interkulturalität führt nicht zum Vergessen oder zur Fusion der vorhandenen Kulturen. Die vorhandenen Kulturen verlieren ihre Wurzeln nicht, aber sie schaffen von ihnen ausgehend neue Möglichkeiten.

Die Anerkennung der Plurinationalität ist ein politisches Mandat für die Förderung der Interkulturalität. Ihre Praxis über einen längeren Zeitpunkt hinweg wird den Ursprung für eine neue Form der Mestizisierung legen. Die koloniale Mestizisierung ist eine entfremdete Mestizisierung, die nicht selten gewalttätig und 
unilateral vom „Mestizisierenden“ reguliert wird. Die postkoloniale Mestizisie-

rung - die sich noch im Stadium eines Projektes befindet-ist im Dialog begründet und plurilateral, sowohl hinsichtlich ihrer Herausbildung als auch ihres Resultats. Die Idee des/der weißen Mestiz_in, die substanziell zur Isolierung der indigenen, bäuerlichen und afro-amerikanischen Bewegungen beigetragen hat, wird schrittweise rückverwandelt und rückübersetzt in Indo-Mestiz_in oder Afro-Mestiz_in, was die kulturellen Identitäten und die Prozesse der Allianzbildung signifikant verändern wird.

\section{Dekolonisierender Feminismus und die Neugründung des Staates}

Der Feminismus hat entscheidend zur Kritik an der dominanten eurozentrischen Epistemologie beigetragen. ${ }^{23}$ Der postkoloniale oder dekolonisierende Feminismus ist von übergreifender Bedeutung bei der Konstruktion einer Epistemologie des Südens, der Interkulturalität und der Plurinationalität - eine Tatsache, die bisher nicht die gebührende Beachtung erfahren hat. Unter dem Begriff des postkolonialen Feminismus verstehe ich die Gesamtheit der feministischen Perspektiven, die 1) die geschlechtsspezifische Diskriminierung im Rahmen eines weiteren Systems der Dominanz und Ungleichheit in heutigen Gesellschaften integrieren, darunter Rassismus und Klassenherrschaft; 2) das Ziel der Dekolonisierung der eurozentrischen Strömungen des Feminismus verfolgen, die in den letzten Jahrzehnten und teilweise bis heute dominant waren bzw. sind; 3) ihren kritischen Blick auch auf die eigene Diversität richten, indem sie Formen der Diskriminierung von Frauen in unterdrückten Gemeinschaften thematisieren und die Diversität innerhalb der Diversität betonen. ${ }^{24}$ Der postkoloniale Feminismus hat noch keine Theorie der Neugründung des interkulturellen und plurinationalen Staates geschaffen, aber einige Aspekte seines möglichen Beitrages dazu sollen hier vorgestellt werden.

Postkoloniale Feminist_innen weisen auf den akkumulativen Charakter von Ungleichheiten hin. Der moderne monokulturelle Staat geht jedoch von der formellrechtlichen Gleichheit der Bürger_innen aus: Da die Unterschiede zwischen ihnen vielfältig sind und sich nicht akkumulieren, können sie dem System zufolge ignoriert werden. Der eurozentrische Feminismus fokussierte in diesem Sinne lediglich auf die Gender-Ungleichheit, so als ob andere Formen der Ungleichheit die monokulturellen Staaten nicht prägen würden. Dadurch essenzialisierte er das Frau-Sein und verdeckte enorme Ungleichheiten zwischen Frauen. Der postkoloniale Feminismus hingegen betont den akkumulativen Charakter von Ungleichheiten und distanziert sich so von der problematischen eurozentrischen Tradition. Er verleiht dem interkulturellen und plurinationalen Staat damit seine tiefere dekolonisierende und antikapitalistische Bedeutung.

Die Kämpfe der indigenen und afro-amerikanischen Frauen haben den lateinamerikanischen Mythos der Demokratie am deutlichsten kritisiert, da sie als schwarze oder indigene Frauen die negativen Auswirkungen des Rassismus am

23 Santos, Toward a New Common Sense: Law, Science and Politics in the Paradigmatic Transition, 1995, S. 32-33.

24 Anzaldúa, Borderlands/La Frontera. The New Mestiza, 1987; Crenshaw, Mapping the Margins: Intersectionality, Identity Politics and Violence against Women of Color, in: Stanford Law Review, 1991; dies., Playing Race Cards: Constructing a Pro-active Defense of Affirmative Action, in: National Black Law Journal, 2000; Curiel, Identidades esencialistas o construcción de identidades políticas: El dilema de las feministas negras, in: Otras Miradas 2002; Suárez Navaz/Hernández Castillo (Hrsg.), Descolonizando el Feminismo, 2008; Vargas, Repensar América Latina desde los retos que traen las diversidades feministas, Vortrag, V Conferencia Latinoamericana y Caribeña de Ciencias Sociales, Cochabamba, 7.-10. Oktober 2009; Harding, Sciences from below: feminisms, postcolonialities, and modernities, 2008. 
stärksten gespürt haben. Ihre Beiträge sind von zweierlei Art. Erstens thematisieren sie die Diskriminierungen von Frauen innerhalb ihrer vermeintlich homogenen Gemeinschaften. Dieser Beitrag zur Neugründung des plurinationalen Staates ist fundamental, denn er zeigt auf, dass die Unterdrückten gleichzeitig Unterdrücker sein können und dass angenommene Konsensentscheidungen in einigen Fällen nicht mehr als selektive Visionen von kollektiven Rechten sind, bei denen die kollektiven und individuellen Rechte von Frauen außen vor bleiben. Dabei zeigen sie auch auf, dass so genannte traditionelle Systeme nicht statisch, sondern dynamisch sind und sich gemäß ihrer eigenen Logik, Rhythmus und Zeit verändern.

Der dekolonisierende Feminismus macht zweitens darauf aufmerksam, dass es nicht eine einzige und universelle Form der Gender-Gleichheit gibt. Innerhalb der indigenen Weltanschauung, bei den Quechua und Aymara, werden die Beziehungen zwischen Mann und Frau als chacha-warmi konzipiert. Die zentrale Idee hinter diesem Konzept ist, dass weder der Mann noch die Frau allein vollkommene Bürger_innen in ihrer Gemeinschaft sind. Sie sind die Hälfte eines Ganzen, und gemeinsam stellen sie ein komplettes Wesen vor der Gemeinschaft dar. Die Aymara-Intellektuelle und Aktivistin María Eugenia Choque Quispe stellte bezüglich der Idee des chacha-warmi kritisch fest: „Diese Vision ist Teil eines andinen Essentialismus, der die alltägliche Realität der Menschen verkennt. " 25 In der Praxis kann die Komplementarität bedeuten, dass zwar die ökonomische Relevanz der Frauen anerkannt wird, nicht aber ihre politische; oder sie kann eine symbolische Anerkennung der Frau bedeuten, verbunden mit ihrer passiven Rolle im öffentlichen Leben. Choque Quispe zufolge sei es nicht Aufgabe indigener Frauen, das Konzept des chacha-warmi zur Gänze zu verwerfen, sondern es neu zu definieren. Das Ziel müsse es sein, das tatsächlich hierarchische Verhältnis, versteckt hinter der Idee der Komplementarität, sichtbar zu machen. Dies ist die Alternative, um die eigene Kultur zu transformieren und gleichzeitig das politisch-kulturelle Erbe des globalen feministischen Kampfes anzureichern, das bisher von eurozentrischen und liberalen Konzepten dominiert ist.

\section{Der experimentelle Staat}

Der historische Prozess der Neugründung des Staates lässt sich wohl am besten anhand seines experimentellen Charakters beschreiben. Der fundamentalste Bruch mit dem modernen eurozentrischen Konstitutionalismus liegt tatsächlich in der Kreation eines experimentellen Staates. Der Prozess der Neugründung hat keine präzise Orientierung und entwickelt sich nicht immer in die Richtung, die sich die Bürger_innen und Völker vorgestellt hatten. Es gibt keine Patentrezepte im Stil von one-size-fits-all, die der moderne Liberalismus so liebt. Es ist nicht möglich, alle Fragen zu lösen oder Fehlentwicklungen eines transformierenden Konstitutionalismus von unten zu vermeiden. Einige neu aufgeworfene Fragen werden wohl von zukünftigen verfassunggebenden Versammlungen zu diskutieren sein.

Der Experimentalismus kann zwei Formen annehmen: die reflexive und die nicht-reflexive. Die reflexive Form beruht auf der Annahme, dass die geschaffenen Institutionen unvollständig sind und die neuen Gesetze eine kurze Lebensdauer haben. Das würde bedeuten, dass die Innovationen permanent von unabhängigen Forschungsinstitutionen überprüft und evaluiert werden, die regelmäßige Berichte über deren Entwicklungen veröffentlichen und am Ende der expe- 
rimentellen Periode neue Debatten geführt und politische Entscheidungen getroffen werden, um das Profil der Institutionen und Gesetze zu beschließen. Der nicht-reflexive Experimentalismus wiederum resultiert aus immer wieder unterbrochenen und widersprüchlichen politischen Praktiken, ohne eine eigene politische Form anzunehmen.

Der experimentelle Staat ist die radikalste Herausforderung für den modernen Staat, dessen Institutionen und Gesetze wie in Stein gemeißelt erscheinen. Der Prozess der Neugründung des Staates ist höchst konfliktreich, und seine langfristige Weiterentwicklung wird davon abhängen, ob sich die verschiedenen Konfliktachsen (ethnisch, regional, klassenspezifisch, kulturell) akkumulieren oder vielmehr neutralisieren werden.

Der Experimentalismus schafft eine politisch mehrdeutige Semantik, bei der es keine definitiven Sieger und Verlierer gibt. Auf diese Weise ermöglicht er eine relative Aufhebung der Konflikte und folglich eine politische Phase, die notwendig sein kann, um Polarisierungen zu verringern. In dieser politischen Wirksamkeit liegt die instrumentelle Dimension des experimentellen Staates. Dennoch sollte sich seine Verteidigung auf Prinzipien stützen, die es den Bürger_innen ermöglicht, die konstituierende Kraft aufrecht zu erhalten, solange die Experimente anhalten und Entscheidungen über konkrete Veränderungen getroffen werden. Es handelt sich somit beim experimentellen Staat um eine längerfristige Spannung zwischen dem Konstituierten und den Konstituierenden.

\section{Menschenrechtsschutz}

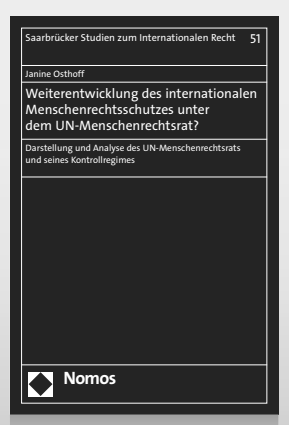

Weiterentwicklung des internationalen Menschenrechtsschutzes unter dem UN-Menschenrechtsrat?

Darstellung und Analyse des UN-Menschenrechtsrats und seines Kontrollregimes Von RAin Dr. Janine Osthoff 2012, 215 S., brosch., 49,- $€$ ISBN 978-3-8329-6985-1

(Saarbrücker Studien zum Internationalen Recht, Bd. 51)

Weitere Informationen: www.nomos-shop.de/14134

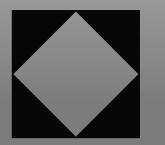

